\title{
FMS PERFORMANCE UNDER BALANCING MACHINE WORKLOAD AND MINIMIZING PART MOVEMENT RULES
}

\author{
Pereira, A. \\ ISEG - Technical University of Lisbon, Rua Miguel Lúpi, 20, 1249-078 Lisboa, Portugal \\ E-Mail: albper@iseg.utl.pt
}

\begin{abstract}
A simulation model is developed to evaluate the performance of a flexible manufacturing system with respect to time in system. The new rule to assign parts to machine-tools we propose, OOM (Only One Machine), designed to minimize parts movements performs poorer than WINQ (Work In Queue), a rule directed at balancing machine workload. Different numbers of automated guided vehicles (AGV) produce significantly different results with the best performance resulting with five AGVs. Three AGVs are too few to handle the transportation requirements, whereas seven may, to some extent, increase AGV blockage. The number of parts that can be entirely processed on one single machine is found to impact performance, but the impact is not consistent across the experimental conditions. Three rules to sequence parts to be processed are found to have a moderate impact when OOM assignment is employed, but have no impact under the WINQ assignment rule.

(Received in October 2010, accepted in April 2011. This paper was with the author 1 month for 1 revision.)
\end{abstract}

Key Words: Flexible Manufacturing System, OOM, Simulation, Time in System, WINQ

\section{INTRODUCTION}

Contemporary market requirements drive the manufacturing into developing capabilities to cope with large product variety, low quantity, small batches and on-time delivery. Flexible manufacturing system (FMS) is a manufacturing setting designed to provide responses to those requirements. Time spent to manufacture a part is therefore critical. Planning and scheduling functions need to be addressed with much greater rigor and precision in order to minimize time in system to process a part. FMSs are agile, provide wide flexibility and are well suited for simultaneous production of a wide variety of part types in low volumes [1].

The main purpose of this research is to evaluate the performance of a dedicated FMS with respect to time in system (T). Two rules to assign machines to parts, three rules to sequence jobs at a particular machine, three levels of available automated guided vehicles (AGV) and two levels of machine commonality will be tested. In a manner similar to the component commonality concept [2], in this study machine commonality refers to the number of parts that can be entirely processed on one single machine. Parts will be loaded onto a pallet in accordance with the first-come-first-served discipline. Once loaded, there is a need to assign the part to the machine-tool (MT) required to perform the next operation. The assignment decision will be based upon one of the two assignment (A) rules: OOM (Only One Machine) and WINQ (Work In Queue). Following the machine assignment decision, parts will be launched into the FMS and scheduled in front of the MT to be processed based upon one of three sequencing $(S)$ rules: shortest processing time (SPT), SPT divided by total processing time (SPT/TOT), and most operations per part (MOPP). Because the performance of a FMS has been shown to be related to the configuration and hardware available to move parts around the system, the number $(V)$ of AGV will be defined as the third experimental factor and will be set at three different levels of intensity: 3, 5 and 7 AGVs. Finally, the number of 
parts that can be entirely processed on one single machine, machine commonality $(M)$, is set at two levels: seven parts out of a total of ten can be entirely processed on one single machine (high level), and three out of ten parts (low level). The performance will then be evaluated across thirty-six different configurations.

\section{LITERATURE REVIEW}

Over the past decades, considerable research effort has been devoted to the domain of manufacturing systems scheduling. In recent years however, attention has been directed towards planning and scheduling problems concerning FMSs [3].

FMS management requires the optimization of several components that can be classified into two groups: design or structural problems and operational problems [4-8]. The first group is concerned with the optimal selection of all the FMS components involving strategic decisions concerning the FMS hardware to meet the user goals and requirements; the second group deals with tactical and control decisions problems such as process planning, machine grouping, part type selection, resource allocation and loading [4]. Shang and Sueyoshi [9] developed a structured framework to support management's deliberation on issues of FMS design and planning specifically intended to assist managers in selecting the most appropriate FMS design.

Caprihan and Wadhwa [3] simulated a hypothetical semi-automated FMS with six flexible machines to measure performance with respect to makespan, the time required to completely process all required parts of all types. Using a Taguchi experimental design with 5 factors (routing flexibility, dispatching rule, sequencing rule, number of pallets, and information delay) each set at 5 levels, they show information delay and number of pallets are significant in terms of their effect on makespan. Routing flexibility, dispatching rule and sequencing rule appear to be relatively less significant. Reddy and Rao [10] developed a hybrid genetic algorithm to address the combined machine and vehicle-scheduling problem to generate an optimum machine and vehicle schedule that is best with respect to the makespan, mean flow time and mean tardiness. Iwata et al. [11] provided a heuristic approach using a decision rule to obtain a good feasible solution for a practical large-scale problem. Three proposed decision rules were investigated through a case study with respect to makespan, mean flow time, mean utilization of machine tools, and mean utilization of transport devices. Shanker and Tzen [12] simulated a FMS to compare the solutions of loading given by mixed integer programming and heuristics methods with respect to CPU time, unbalance and number of tool slots used, and to investigate the effect of loading policies in conjunction with four dispatching rules on system performance as measured by machine utilization. Ozden [13] conducted a simulation study of multiple-load-carrying AGV in a FMS whose performance was measured with respect to throughput, average utilization of the machining station, and average utilization of the AGVs. Kuzgunkaya and ElMaraghy [14] present a fuzzy multi-objective mixed integer optimization model to evaluate reconfigurable manufacturing system investments used in a multiple product demand environment and compare the performance of flexible and reconfigurable systems. Singh [15] addresses the multi criteria dynamic scheduling problem with machine breakdowns in a flexible job shop.

\section{OOM AND WINQ}

OOM is a new rule proposed in this study. OOM attempts to assign to each incoming part a machine that can perform all the operations required by the part. If this machine is not available at the time it is needed or if such a machine does not exist, OOM then attempts to assign a machine that can complete the next largest number of consecutive operations 
required by the part. This process continues until a machine that can process at least one operation is found. In the event that more than one machine is found to be available, OOM randomly selects the machine to assign to the part. If no machine is found to be available, the incoming part is routed to a parking area where it waits until any machine becomes available.

The WINQ rule assigns machines to incoming parts based on the current total processing time allocated to the parts located in the queue preceding the machine. A part requesting processing for a particular operation will cause WINQ to scan the machines for one capable of performing the requested operation with at least one spot available in its input queue. Those meeting this requirement are placed in a temporary candidate machine set. If two or more machines are placed in the set, WINQ assigns the machine with the current lowest total processing time requirement reflected in its input queue. If no candidate machine can be selected, WINQ parks the part requesting processing in a waiting area until any machine becomes available.

\section{THE FLEXIBLE MANUFACTURING SYSTEM}

A FMS can be defined as a system composed of CNC machines, an automated material handling system, and a computer-controlled network that coordinates the activities of processing stations and the material-handling system [8].

The FMS to be studied produces ten parts on five identical MT. Each machine is able to process only certain part types based upon the tools that are available in that machine's tool magazine. A survey of experts in manufacturing reveals a comparatively smooth distribution of FMS size between two and ten stations and it appears that for most manufacturing applications, ten stations or fewer seem to be adequate [16]. Caprihan and Wadhwa [3] and Chen and Chung [17] studied flexible manufacturing systems comprised of six and four machines, respectively. A load/unload work centre is located beside the machine-tools area. The parts to be manufactured arrive to the system as raw work pieces and are loaded onto pallets at the load/unload work centre. Part arrival is described by a uniform distribution. Upon arrival, parts are loaded onto pallets. The loading takes place on a first-come-firstserved basis, and as soon as a pallet becomes available in the load/unload station. The time to load a part onto a pallet is uniformly distributed between 0.75 and 1.0 minute.

Various operations are performed to complete the finished part. Depending upon the part type, the required number of operations varies between two and four $[3,17,18]$. The number of operations needed to complete a part is always greater than or equal to the number of machines visited by the part, because some machines have the capability of processing two or more consecutive operations required by a part. The five machines, however, do not have identical capabilities because the tools assigned to each machine prior to starting the production process are not equivalent. Within the same machine, the transition between two consecutive operations is solely mediated by the negligible fractional set-up time and, hence, the processing time for consecutive operations in the same machine is aggregated in to one single total processing time. A total of nine operations are performed by the five MT. Pallets holding parts to be manufactured are moved throughout the FMS by means of an AGV system. Each machine is preceded by an input queue. When the pallet arrives before the queue in front of the machine, a transfer mechanism is activated by the control computer and the pallet is shifted from the AGV onto the on-shuttle. The on-shuttle has the capability of rearranging work-parts for processing according to the sequencing priority. Work parts entering the queue preceding the machine-tools are placed ahead of previously placed work parts with lower sequencing priority [19]. The AGV is then released to cruise along the guided path until a new request is issued. If the MT is busy, the part requiring processing waits in the input queue until the machine becomes available. 
Processing begins as soon as the MT becomes available. Once processed, the part is moved to the output queue following the MT to wait for a free transporter to collect and carry it to the next destination (MT or unload). If, when a machine finishes processing a part, there is no available space in the output queue to which the part can be moved, the machine is blocked and is unable to process the next part. Both the input and the output queues have a capacity of five work-parts and pallets. Previous studies [20-21] have simulated FMS with queues of five.

Completed parts are removed from the pallet at the load/unload work centre before leaving the system. The pallet is then reloaded or stored until needed. The pallets are of the general purpose type and unrestricted, so that whenever a move request is sent to an AGV, a pallet is assumed to be available. The AGV system consists of a variable number of vehicles that move unidirectionally on one of two-railed tracks. One track accommodates forward movement, the other reverse. To change an AGV direction, a special mechanism actuates the crossover links to shift the AGV from one track to the other. Bidirectional movement is allowed along the crossover links between the two main tracks. Sixteen control points are defined along the track. When an AGV reaches a control point, it may receive instructions regarding its movement (load, unload, proceed, wait). During the operations of the system, idle vehicles cruise along the guided path until a request is placed. Fig. 1 shows the dedicated FMS simulated in this study. A similar layout is found in Reddy and Rao [10].

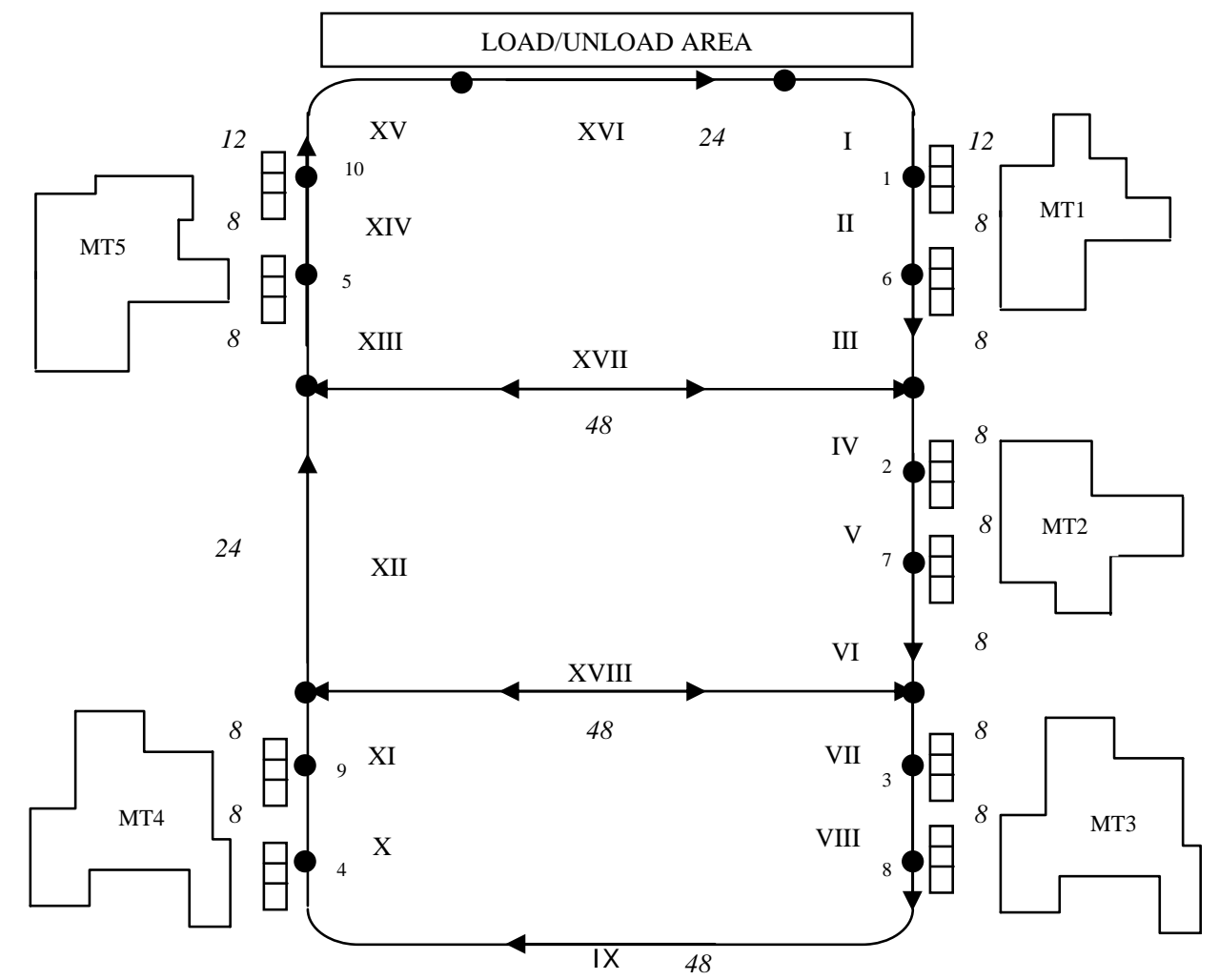
Input/output queue Control point
Direction of AGV flow
Italics indicate distance (m)
Arabic numerals indicate control point numbers
Roman numerals indicate segment number

Figure 1: The Flexible Manufacturing System.

Two groups of 10 parts are defined. Seven out of the ten parts of the first group can be entirely processed on one single machine, whereas in the second group only three can be entirely processed on one single machine. We call the first, high machine commonality group, 
and the second is termed low machine commonality group. The first group corresponds to the high level of machine commonality experimental factor, the second to the low level. A previous study [22] used commonality as an experimental factor set at two levels, high and low. An entry of 1 in the bottom portion of Table I indicates the operation in that column can be performed by the machine. The top portion shows the time (in minutes) to process the operations required by the part. For each part, operations are processed in the sequence shown, from left to right. Part name is followed by an indication to which group it belongs: high machine commonality (h), or low (l). Five parts are common to both groups. Total processing time over both groups is the same.

Table I: Operations required to manufacture all parts.

\begin{tabular}{|llllllllll|}
\hline & \multicolumn{8}{c|}{ Operation } \\
\cline { 2 - 9 } Part & 1 & 2 & 3 & 4 & 5 & 6 & 7 & 8 & 9 \\
\hline A $(h, l)$ & 1 & 2 & 6 & & & & & & \\
B (h) & 3 & 5 & & 2 & & & & & \\
C $(h, l)$ & & 2 & 2 & 1 & & & & & \\
D (h) & & & 6 & 5 & 4 & 1 & & & \\
E $(h, l)$ & & & & 2 & & 8 & & & \\
F (h, l) & & & & & 9 & 4 & & & \\
G (h) & & & & & & & 5 & 1 & 4 \\
H (h, l) & & & & & & & 3 & 7 & \\
I (h) & & & & & 3 & & 5 & & 1 \\
J (h) & & & & & 6 & & 1 & 1 & \\
K (l) & 3 & 5 & & & & & 2 & & \\
L (l) & & & & 6 & 5 & 4 & & 1 & \\
M (l) & & & & 5 & & & & 1 & 4 \\
N (l) & & & & 3 & & & 5 & & 1 \\
O (l) & & & 6 & & 1 & & 1 & & \\
\hline
\end{tabular}

\begin{tabular}{|llllllllll|}
\hline Machine & & 1 & & 1 & & & & & \\
\hline 1 & 1 & & 1 & & 1 & & & & \\
2 & 1 & 1 & & 1 & & 1 & 1 & & \\
3 & 1 & & & & & 1 & 1 & 1 & 1 \\
4 & & & & & & 1 & 1 & & 1 \\
5 & & 1 &
\end{tabular}

It is felt that keeping the total processing time constant over the two groups and an equal number of parts are preferable to maintaining a fixed set of ten parts and changing the machine environment by altering the set of tools assigned to each machine loaded into the MT magazine. A change in tool assignment might significantly affect the relative operating characteristics of the five machines, thereby reducing our ability to compare results across the machine commonality factor setting. It is possible to route each part through the system in several different ways. A total of 120 and 131 different routings are possible for the high and low commonality product groups, respectively. The number of routings for any part $p$ is:

$$
\prod_{j=1}^{9}\left(O_{p j} \times M_{j}\right) \quad \forall O_{p j} \neq 0
$$

where:

$O_{p j} \quad$ - is set equal to 1 if part $p$ requires operation $j$, and set equal to 0 otherwise.

$M_{j} \quad$ - is the number of machines that process operation $j$. 
For example, for part $\mathrm{B}(p=\mathrm{B})$, using (1), the number of routings is found to be equal to 12. The complete enumeration of the routings for part B, as indicated in Table II, shows that some of the routes are dominated by others and may be dropped from further consideration. Routing 1, for example, dominates routes 6,8 and 10. Because of the dominance effect, the total of 120 and 131 routings is reduced to 56 and 58, respectively.

Table II: Routings for part B.

\begin{tabular}{|crrrrrrrrrrrr|}
\hline & \multicolumn{110}{|c|}{ Opeute } \\
\cline { 2 - 13 } & 1 & 2 & 3 & 4 & 5 & 6 & 7 & 8 & 9 & 10 & 11 & 12 \\
\hline 1 & 3 & 1 & 5 & 5 & 1 & 3 & 1 & 3 & 1 & 3 & 5 & 5 \\
2 & 3 & 2 & 3 & 2 & 3 & 2 & 2 & 2 & 3 & 3 & 3 & 2 \\
4 & 3 & 2 & 3 & 2 & 3 & 2 & 3 & 3 & 2 & 2 & 2 & 3 \\
\hline
\end{tabular}

The input variables for this study and their associated distributions and parameters are presented in Table III and have been found in previous studies [11-13; 23-24].

Table III: Flexible manufacturing system operational data.

\begin{tabular}{|ll|ll|}
\hline Number of identical machines & 5 & Time to un/load AGV & 0.3 minutes \\
Number of parts & 10 & AGV (loaded) speed/min & $246.06 \mathrm{ft}(75 \mathrm{~m})$ \\
Average shop loading & $70 \%$ & AGV (empty) speed/min & $255.90 \mathrm{ft}(78 \mathrm{~m})$ \\
Number of pallets & $\sim$ & Disposition of idle AGVs & Cruise \\
Number of AGVs & $3,5,7$ & Initial location of AGVs & Variable \\
Parts inter-arrival time & Uniform & Number of control points & 16 \\
Input/output queue capacity & 5 & Number of segments & 16 \\
\hline
\end{tabular}

Using a uniform distribution for parts inter-arrival with mean of 2.857 minutes, this research seeks 70 per cent average machine utilization over the simulation horizon, midway within the range from 52 to 88 per cent reported in the literature [12, 13, 25]. Time to load or unload an AGV is short as a result of automation. Descriptions of actual systems report load/unload cycle times as low as five seconds [26]. Sabuncuoglu and Hommertzheim [21] used 0.3 minutes to load/unload the AGVs. Elsayed and Boucher [27] have reported AGV speeds varying from 200 to $260 \mathrm{ft} / \mathrm{min}$.

Tools required for the entire sets of operations performed by any machine have been loaded onto the tool magazine of each MT. Machine-tools are assumed to work without breakdowns. The manufacturing environment to be studied is consistent with respect to the hardware specifications and operational parameters [12, 13, 21, 25, 28]. This study addresses the research hypotheses listed in Table IV.

\section{SIMULATION MODEL}

A simulation model is developed to address the research hypotheses. There is an increasing trend towards the use of simulation to evaluate manufacturing systems. The trend results, in part, from the fact that automated systems are complex, and are therefore difficult to model mathematically [9]. The early work in simulation and that which has been dominant in management science and operations research over the history is system analysis, where the intent is to mimic behaviour to understand or improve system performance [28]. Advantages of simulation in manufacturing have been reported [29-30]. For the modelling purpose in this paper, Visual SLAM and AweSim were used in the modelling of the FMS [31]. SLAM provides a simulation language that allows alternative approaches to modelling by easily 
altering parameters so that many variations of a system can be analysed. It has the ability to allow the modeller to insert $C$ language subroutines where required [32-33]. Legato and Mazza [34], used Visual SLAM language to simulate a queuing network model. Dhouib et al. [35] used AweSim/VisualSlam to measure the effective throughput of non-homogeneous automatic transfer lines. Other studies relied on simulation as well [3, 24].

The code developed to model the system consists of two major parts. The first part is coded using standard SLAM network statements; the second part is coded in C to perform specialized logic requirements not provided by the standard SLAM network nodes. Control statements to monitor the crossing of state variables against a threshold, servers, physical characteristics for the AGVs, the flow of entities through a series of regular and service activities, logic and decision nodes, and rules to stop the simulation and to collect statistical observations are defined. The bulk of the $\mathrm{C}$ code develops the logic associated with both OOM and WINQ assignment rules. Other subroutines are developed to access the attributes of the parts in the output queue following the MT to facilitate a decision to request a vehicle from the AGV fleet and to reroute the part to the next available machine to perform the next operation, or to request a vehicle to transport the part to the unloading area to exit the system. A third group of subroutines were written to release into the shop parts residing in the parking area as the result of the temporary unavailability of input queue space. Each MT is modelled as a one server service activity. These service activities are preceded and followed by queue nodes to model the input and output buffers. A block feature is inserted between the server activity and the output buffer to block the server whenever a part finishing processing seeks a spot in the output buffer when it is at capacity. Parts are created at the load/unload area according to a uniform distribution. Each part carries a set of 12 attributes to define characteristics such as creation time, part type or number of required operations. Attribute value can be defined as a constant; it may vary as the part flows through the network, or it may be set as binary (0-1) to define a mutually exclusive condition. In this study, for example, attribute 4 is binary where 1 means the part passed through an initial counter.

Following their creation, parts flow through a counter which is incremented to an upper limit representing the number of parts of interest. Each part flowing through the counter increases it by one unit. The current counter value is then compared to the upper limit. When the counter reaches the upper limit, the counter is disabled by making the parts bypass it. Parts passing through the counter are assigned a value of 1 to attribute 4; parts bypassing it carry a value of zero in attribute 4 . A second counter at the very end of the network is incremented by 1 whenever a part having attribute 4 set equal to 1 passes through it. The simulation run stops when this second counter reaches the value that matches the upper limit set for the first counter. A sufficient large time is allocated for each simulation run to prevent the simulation from stopping when current simulation time is greater than or equal to the ending time specified for the simulation. This ensures that the simulation will terminate when the last part that passed through the first counter leaves the system. Both the discrimination of parts based on the value of attribute 4 and the stopping rule guarantees that all parts of interest will be accounted for statistical purposes. Lack of a monitoring device of this sort would greatly bias the time in system statistics. Similar precautions have been implemented in previous studies [21, 31]. A MT will be assigned to the part based upon the current assignment rule, OOM or WINQ. A SLAM global variable emulates a toggle switch so that the selection of OOM as the current assignment rule prevents the computer program from executing the WINQ subroutine and vice versa. The current assignment rule subroutine is called either by a new part entering the shop or by an in-process part seeking a MT to process the next operation(s). Following the execution of the assignment rule subroutine, the control is returned back to the SLAM network and the part is placed in the input queue or in the parking area. Detect nodes monitor the number of parts in the input queues so that a part residing at the parking area can seize a 
spot in the input queue as soon as it becomes available. Upon completion, parts pass through statistical collection nodes and leave the system. Statistics are collected with the system running under steady state condition.

The validity of the model was measured using several procedures. As each segment of coding was added or changed its internal logic was tested to confirm that it functioned in the manner intended. Several runs were performed using predetermined parameters in the anticipation that only part of the code would be executed and saturated while leaving unrelated portions of the code unexecuted. Additionally, several runs using constant values were performed and the output was compared to that obtained using a desk calculator and intuition. A trace of the simulation was performed to "visualize" both the flow of entities throughout the nodes and the flow of the AGV along the guided path. At specified simulation times some runs were deliberately terminated and file contents were printed to check for accuracy of the entities' attributes. To complement the error detection features provided by SLAM, additional measures were coded either to prevent entities from taking prohibited routings or from engaging in extraneous actions. Error warnings were generated only when deliberately induced. Observations confirmed the model was performing as intended.

\section{RESULTS}

\subsection{Pilot study}

A pilot study is conducted to identify and evaluate the transient period with respect to time in system. The reduction of point estimator bias, which is caused by using artificial initial conditions, as well as the determination of the end of the transient period is of paramount importance and is well documented [36]. All four configurations selected to run the pilot study have 3 AGVs, since demand on automated vehicles it is expected to be the most stringent at the lowest level of number of vehicles in the system. For each configuration, five replications are compared. Each replication is run separately under the same empty and idle initial conditions but with different random number streams. For each replication, statistical results are gathered based upon the number of parts actually processed, beginning at the output level of 400 completed parts and ending at the output level of 2,000 completed parts in increments of 100 output units.

Determination of the steady state is made by both visual inspection and statistical analysis. A total of eight plots of the response variables against the number of processed parts are generated for the four configurations. Visual inspection shows the steady state is reached when 600 parts are processed. Observations from 600 through 2,000 processed parts are used to test the null hypothesis that the means for time in system from 600 through 2,000 processed parts are equal. Results indicate that there is not sufficient evidence to reject the null hypotheses.

In addition, three treatment combinations were randomly selected and simulated for an extremely long run length until 15,000 parts have been completely processed. Average differences of 0.85 per cent are found when the results are compared to those obtained for smaller steady state runs. The simulated shop is stable and does not generate increasingly longer queues.

\subsection{Main study}

Eight replications per simulation run configuration are necessary in order to obtain a 95 per cent confidence interval for the sample mean based on the following [31]:

$$
I=\left[\left(t_{\alpha / 2, I-1} \times s_{y}\right) / g\right]^{2}
$$


where:

I - number of required replications for the study.

$t_{\alpha / 2, I-1} \quad$ - value from the table of the $t$-statistic with $I-1$ degrees of freedom.

$s_{y} \quad$ - estimate of the standard deviation of the response variable.

$g \quad$-half-width of the desired confidence interval.

One week of seven days, 24 hours per day, is used as the observation period. Run lengths of the same magnitude or less were used in previous flexible manufacturing simulation studies [12, 13, 19, 20, 37]. The inter-arrival distribution generates a mean of 21 parts per hour, therefore it is estimated that approximately 3,500 parts enter the system over the one week time span. The observation period for each replication is set at 3,500 processed parts with data gathered for this number of parts processed following the establishment of the steady state. After reaching the steady state, statistical arrays are cleared while leaving the state of simulated system intact.

The main study consists of a full factorial model of all four factors simulated for eight replications. The response variable, time in system, is collected for the 3,500 completed parts. The total output is made up of 288 observations. Within each of the 36 cells, normal cumulative probability plots are generated for the response variable. In each plot, the horizontal coordinate represents the expected value of the residuals and the vertical coordinate represents the ordered residuals [38]. The plots show no major departures from normality. Linearity is supported by the high coefficient of correlation between the ordered residuals and their expected values under normality. The lowest coefficient of correlation for time in system is equal to 0.8429 . Since no major departures from normality were found and because all $n_{i}=8$ ( $i=1,2, \ldots, 36$ ), the model I (fixed factor levels) of the analysis of variance (ANOVA) [39] will be used to analyse the data. Average machine utilization approaches 70 per cent over all experimental conditions ranging between 69.74 per cent and 69.80 per cent. The factor settings do not significantly affect the overall shop utilization.

Table IV: ANOVA for time in system.

\begin{tabular}{|c|c|c|c|c|c|}
\hline Source & \multicolumn{2}{|c|}{ Sum of squares } & Mean Squares & F ratio & $\operatorname{Pr}>F$ \\
\hline Between & \multicolumn{2}{|c|}{16631.07332778} & 475.17352365 & \multirow[t]{2}{*}{611.60} & 0.0000 \\
\hline Within & 195. & 30000 & 0.77693175 & & \\
\hline Total & 16826. & 2778 & & & \\
\hline Hypothesis & Source & $d f$ & ANOVA SS & $F$ value & $\operatorname{Pr}>F$ \\
\hline $\mathrm{Ho}(1): T_{A}=0$ & $A$ & 1 & 13674.9965680 & 17601.28 & $0.0000 *$ \\
\hline $\mathrm{Ho}(2): T_{V}=0$ & $V$ & 2 & 1664.4251548 & 1071.15 & $0.0000 *$ \\
\hline $\mathrm{Ho}(3): T_{M}=0$ & $M$ & 1 & 40.9814222 & 52.75 & $0.0001 *$ \\
\hline $\mathrm{Ho}(4): T_{S}=0$ & $S$ & 2 & 33.4219590 & 21.51 & $0.0001 *$ \\
\hline $\mathrm{Ho}(5): T_{A V}=0$ & $A \times V$ & 2 & 626.4117173 & 403.13 & $0.0000 *$ \\
\hline $\mathrm{Ho}(6): T_{M A}=0$ & $M \times A$ & 1 & 209.5445680 & 269.71 & $0.0001 *$ \\
\hline $\mathrm{Ho}(7): T_{A S}=0$ & $A \times S$ & 2 & 20.8018756 & 13.39 & $0.0001 *$ \\
\hline $\mathrm{Ho}(8): T_{M V}=0$ & $M \times V$ & 2 & 227.3948256 & 146.34 & $0.0001 *$ \\
\hline $\mathrm{Ho}(9): T_{V S}=0$ & $V \times S$ & 4 & 10.8190201 & 3.48 & $0.0087 *$ \\
\hline $\mathrm{Ho}(10): T_{M S}=0$ & $M \times S$ & 2 & 1.9464751 & 1.25 & 0.2875 \\
\hline $\mathrm{Ho}(11): T_{M A V}=0$ & $M \times A \times V$ & 2 & 111.6330882 & 71.84 & $0.0001 *$ \\
\hline $\mathrm{Ho}(12): T_{A V S}=0$ & $A \times V \times S$ & 4 & 6.0155076 & 1.94 & 0.1050 \\
\hline $\mathrm{Ho}(13): T_{M V S}=0$ & $M \times V \times S$ & 4 & 1.4754368 & 0.47 & 0.7543 \\
\hline $\mathrm{Ho}(14): T_{M A S}=0$ & $M \times A \times S$ & 2 & 0.3962965 & 0.26 & 0.7751 \\
\hline $\mathrm{Ho}(15): T_{M A V S}=0$ & $M \times A \times V \times S$ & 4 & 0.8094159 & 0.26 & 0.9031 \\
\hline
\end{tabular}

* significant at $\alpha=0.01$ 
Time in system is less when parts were assigned to machine using the WINQ rule rather than OOM. Different numbers of AGVs produce significantly different results, with the best performance resulting from the $5 \mathrm{AGV}$ setting. Machine commonality is found to impact time in system, but the impact is not consistent across the experimental conditions. Depending upon how other factors are combined, the lowest time in system is found to be associated with both the low and high levels of machine commonality. Sequencing rules are found to have a moderate impact when OOM is employed to assign parts to machines, but the sequencing rules have no impact under the WINQ assignment rule. The analyses of variance results for time in system are displayed in Table IV.

$M \times A \times V$ is the highest order interaction that is statistically significant. We then studied $M \times A$ at all levels of $V ; M \times V$ at all levels of $A$; and $A \times V$ at all levels of $M$. Results show that the WINQ rule performs significantly better than OOM regardless of how machine commonality and number of AGV are set. Table V displays the cell means.

Table V: Mean time in system.

\begin{tabular}{|c|c|c|c|c|}
\hline & \multicolumn{2}{|c|}{ Low machine commonality } & \multicolumn{2}{|c|}{ High Machine Commonality } \\
\hline & OOM & WINO & $\mathrm{OOM}$ & WINO \\
\hline $3 \mathrm{AGV}$ & 42.35 & 36.20 & 42.55 & 29.47 \\
\hline $5 \mathrm{AGV}$ & 39.26 & 24.26 & 40.74 & 23.88 \\
\hline $7 \mathrm{AGV}$ & 40.60 & 25.52 & 41.77 & 25.25 \\
\hline
\end{tabular}

The two best performers combine WINQ and 5 AGVs at either high or low level of machine commonality. Results consistently indicate that time in system is lower when number of vehicles is set at 5, and higher at 3 AGVs. Reductions on time in system approaching $33 \%$ can be achieved by increasing the number of vehicles from 3 to 5 at the low level of machine commonality in combination with WINQ. These results indicate that time in system decreases as the number of vehicles increases from 3 to 5 . However, the addition to the fleet of two more vehicles is not followed by a further improvement on time in system. To the contrary, the addition of 2 more vehicles resulted in an increase in time in system. This suggests that $3 \mathrm{AGV}$ are too few to handle the transportation requirements, whereas 7 AGVs may, to some extent, increase AGV blockage. Time in system consistently decreases as the assignment rule is shifted from OOM to WINQ. A substantial decrease of more than 41 per cent is obtained when the assignment rule is shifted from OOM to WINQ with 5 vehicles in the system at the high level of machine commonality.

Two of the five significant first order interactions, $A \times S$ and $V \times S$, involve the sequencing rule factor ( $S$ ), which happens to be the only main factor not involved in the single significant second order interaction $(M \times A \times V)$. Results show there are significant differences in mean time in system for a given sequencing rule depending on whether OOM or WINQ is employed to assign machines. Differences due to sequencing with SPT, SPT/TOT or MOPP within the WINQ assignment rule are not significant, however. The OOM $\times$ MOPP combination performs poorer than any other combination.

Table VI: Mean time in system.

\begin{tabular}{|rrrrrrr|}
\hline \multicolumn{3}{|c}{ OOM } & & \multicolumn{3}{c|}{ WINQ } \\
\cline { 1 - 3 } \cline { 7 - 8 } SPT/TOT & SPT & MOPP & & SPT/TOT & SPT & MOPP \\
40.75 & 40.81 & 42.07 & & 27.35 & 27.40 & 27.53 \\
\hline
\end{tabular}

Analysis of time in system grouped by $S$ and $V$ shows the lowest times in system are associated with 5 AGVs regardless of the scheduling rule, as shown in Table VII. 
Table VII: Mean time in system.

\begin{tabular}{|crrr|}
\hline AGV & SPT/TOT & SPT & MOPP \\
\hline 3 & 37.15 & 37.28 & 38.51 \\
5 & 31.87 & 31.95 & 32.29 \\
7 & 33.14 & 33.10 & 33.61 \\
\hline
\end{tabular}

The major surprise of the results is the superior performance of WINQ when compared to OOM. Contrary to the expectation of Stecke and Solberg [37], an assignment rule designed to minimize part movement was found to be significantly inferior to an assignment rule directed at balancing machine workload. The fact that OOM consistently performs worse than WINQ can possibly be explained by how machines have been tooled prior to operation of the FMS. At the high level of machine commonality, machines 1 and 5 are not tooled to completely process any of the 10 parts. Machine 4, by itself, can process five of the seven parts that can entirely be processed on a single machine. This fact results in an extremely high demand on machine 4 while leaving machines 1 and 5 excessively idle. At the low level of machine commonality, machines 2, 3 and 4 have the capability to process all operations required by three parts. As a result of the machine tooling, the OOM assignment rule appears to enhance the imbalance, as compared to WINQ, which acted to distribute the workload more evenly across the five machines.

The analysis of the average machine utilization controlled for assignment rule, number of AGVs, and machine commonality shows that for every treatment combination, the WINQ maximum machine utilization were lower than those generated by OOM. In every case, the maximum utilization imbalance associated with WINQ is smaller than the correspondent OOM imbalance. The tendency of OOM to overload machines capable of entirely processing a part is illustrated by the machine 4 utilization when machine commonality is set at high level. At high level, seven out of ten parts can entirely be processed on one machine. Furthermore, machine 4 can process five of these seven parts. Results exhibit a nearly full utilization for machine 4. At the low level of machine commonality, the highest machine utilization levels under OOM are associated with machines 2 and 4, capable to entirely process parts $\mathrm{E}$ and $\mathrm{F}$, and $\mathrm{F}$ and $\mathrm{H}$, respectively. Machine utilization is as high as $98.66 \%$ for machine 2 and $95.03 \%$ for machine 4 . The lesser amount of transport time resulting from the OOM assignment rule more than counteracted by the increased queue delays generated by machine workload imbalances and extremely high maximum utilizations. It is believed that under a different tool assignment aimed at equally distributing the ability to completely process a part, OOM would have performed better. This issue will be further studied in future research. The sequencing factor is found to be statistically significant when combined either with the assignment rule factor or with the number of vehicles factor. The SPT/TOT rule combined with the WINQ assignment rule yields the best time in system performance for the interaction $S \times A$. On the other hand, SPT/TOT scheduling rule combined with $5 \mathrm{AGV}$ yielded the best performance interaction $S \times V$. This suggests that the overall best treatment combination with respect to time in system is the one that assign machines using WINQ, uses 5 AGVs, and sequences parts under SPT/TOT or SPT.

\section{CONCLUSIONS}

This research used simulation to evaluate the performance of a dedicated FMS made up of five machines to process ten different parts. Parts movements among machines were made by mean of a fleet of AGVs. Performance of the system was measured with respect to time in system under thirty-six different configurations. 
It was found that the lowest time in system was provided by the configuration in which parts were assigned to machines using the WINQ rule at the high level of machine commonality and five vehicles used to move parts among machines. Sequencing was found to be significant when combined either with the assignment rule or with the number of vehicles. The overall best treatment combination with respect to time in system is the one that sets the FMS with high machine commonality, operating under WINQ, five AGVs, and SPT/TOT as the scheduling rule. The second best treatment combination only differs from the previous one because it uses SPT as the scheduling rule instead.

Machine utilization was controlled by the time between arrival for the incoming parts and it was manipulated so that an average machine workload of 70 per cent was maintained. In spite of negligible variation across the overall machine utilization, remarkable variation was found among the five machines for both OOM and WINQ. Variation among machines was found to be more levelled under WINQ than under OOM.

\section{REFERENCES}

[1] Udhayakumar, P.; Kumanan, S. (2010). Task scheduling of AGV in FMS using non-traditional optimization techniques, International Journal of Simulation Modelling, Vol. 9, No. 1, 28-39

[2] Mirchandani, P.; Mishra, A. K. (2002). Component commonality: models with product-specific service constraints, Production and Operations Management, Vol. 11, No. 2, 199-215

[3] Caprihan, R.; Wadhwa, S. (2005). Scheduling of FMSs with information delays: A simulation study, International Journal of Flexible Manufacturing Systems, Vol. 17, No. 1, 39-65

[4] Stecke, K. E. (1983). Formulation and solution of nonlinear integer production planning problems for flexible manufacturing systems, Management Science, Vol. 29, No. 3, 273-288

[5] Kusiak, A. (1985). Flexible manufacturing systems: a structural approach, International Journal of Production Research, Vol. 23, No. 6, 1057-1073

[6] Kusiak, A. (1986). Application of operational research models and techniques in flexible manufacturing systems, European Journal of Operational Research, Vol. 24, No. 3, 336-345

[7] Carrie, A.; Perera, D. (1986). Work scheduling in FMS under total availability constraints, International Journal of Production Research, Vol. 24, No. 6, 1299-1308

[8] Sabuncuoglu, I.; Lahmar, M. (2003). An Evaluative Study of Operation Grouping Policies in an FMS, International Journal of Flexible Manufacturing Systems, Vol. 15, No. 3, 217-239

[9] Shang, J.; Sueyoshi, T. (1995). A unified framework for the selection of a Flexible Manufacturing System, European Journal of Operational Research, Vol. 85, No. 2, 297-315

[10] Reddy, B. S. P.; Rao, C. S. P. (2006). A hybrid multi-objective GA for simultaneous scheduling of machines and AGVs in FMS, International Journal of Advanced Manufacturing Technology, Vol. 31, No. 5-6, 602-613

[11] Iwata, K.; Murotsu, A.; Oba, F.; Yasuda, K.; Okamura, K. (1982). Production scheduling of flexible manufacturing systems, CIRP Annals - Manufacturing Technology, Vol. 31, No. 1, 319322

[12] Shanker, K.; Tzen Y-J. (1985). A loading and dispatching problem in a random flexible manufacturing system, International Journal of Production Research, Vol. 23, No. 3, 579-595

[13] Ozden, M. (1988). A simulation study of multiple-load-carrying automated guided vehicles in a flexible manufacturing system, International Journal of Production Research, Vol. 26, No. 8, 1353-1366

[14] Kuzgunkaya, O.; ElMaraghy, H. A. (2007). Economic and strategic perspectives on investing in RMS and FMS, International Journal of Flexible Manufacturing Systems, Vol. 19, No. 3, $217-$ 246

[15] Singh, A. (2010). Aspect of selective rerouting in multicriteria scheduling of flexible manufacturing, International Journal of Simulation Modelling, Vol. 9, No. 3, 131-142

[16] Mehrabi, M. G.; Ulsoy, A. G.; Koren, Y.; Heytler, P. (2002). Trends and perspectives in flexible and reconfigurable manufacturing systems, Journal of Intelligent Manufacturing, Vol. 13, No. 2, 135-146 
[17] Chen, I. J.; Chung, C-S. (1996). Sequential Modeling of the Planning and Scheduling of Flexible Manufacturing Systems, Journal of the Operational Research Society, Vol. 47, 1216-1227

[18] Sridharan, R.; Babu, A. S. (1998). Multi-level scheduling decisions in a class of FMS using simulation based metamodels, Journal of the Operational Research Society, Vol. 49, No. 6, 591602

[19] Chang, Y-L.; Sullivan, R.; Wilson, J. (1986). Using SLAM to design the material handling system of a flexible manufacturing system, International Journal of Production Research, Vol. 24, No. $1,15-26$

[20] Choi, R. H.; Malstrom, E. M. (1988). Evaluation of traditional work scheduling rules in a flexible manufacturing system with a physical simulator, Journal of Manufacturing Systems, Vol. 7, No. $1,33-45$

[21] Sabuncuoglu, I.; Hommertzheim, D. L. (1989). An investigation of machine and AGV scheduling rules in an FMS, Stecke, K. E.; Suri, R. (Eds.), Proceedings of the $3^{\text {rd }}$ ORSA/TIMS Conference on Flexible Manufacturing Systems: Operations research models and applications, 261-266

[22] Günther, H. O.; Gronalt, M.; Zeller, R. (1998). Job sequencing and component set-up on a surface mount placement machine, Production Planning \& Control, Vol. 9, No. 2, 201-211

[23] Sarin, S. C.; Chen, C. S. (1987). The machine loading and tool allocation problem in a flexible manufacturing system, International Journal of Production Research, Vol. 25, No. 7, 1081-1094

[24] Chan, F. (2002). Evaluation of combined dispatching and routing strategies for a flexible manufacturing system, Proceedings of the Institution of Mechanical Engineers, Part B. Journal of Engineering Manufacture, Vol. 216, No. 7, 1033-1046

[25] Denzler, D. R.; Boe, W. J. (1987). Experimental investigation of flexible manufacturing system scheduling decision rules, International Journal of Production Research, Vol. 25, No. 7, 979-994

[26] Miller, R. K.; Subrin, R. (1987). Automated guided vehicles and automated manufacturing, Society of Manufacturing Engineers, Dearborn, Michigan

[27] Elsayed, E. A.; Boucher, T. O. (1985). Analysis and Control of Production Systems, PrenticeHall, Englewood Cliffs, New Jersey

[28] Nance, R.; Sargent, R. (2002). Perspectives on the evolution of simulation, Operations Research, Vol. 50, No. 1, 161-172

[29] Hachicha, W.; Masmoudi, F.; Haddar, M. (2007). An improvement of a cellular manufacturing system design using simulation analysis, International Journal of Simulation Modelling, Vol. 6, No. 4, 193-205

[30] Kehris, E. (2009). Web-based simulation on manufacturing systems, International Journal of Simulation Modelling, Vol. 8, No. 2, 102-113

[31] Pritsker, A. A.; O’Reilly, J. J.; LaVal, D. K. (1997). Simulation with Visual SLAM and AweSim, John Wiley \& Sons, New York

[32] Szczerbicki, E. (2000). Simulation modeling for complex production systems, Cybernetics and Systems: An International Journal, Vol. 31, No. 3, 333-351

[33] Szczerbicki, E.; Williams, J. (2001). Developing agile manufacturing strategy with AWESIM simulation platform, Cybernetics and Systems: An International Journal, Vol. 32, No. 7, 719-736

[34] Legato, P.; Mazza, R. (2001). Berth planning and resources optimization at a container terminal via discrete event simulation, European Journal of Operational Research, Vol. 133, No. 3, 537547

[35] Dhouib, K.; Gharbi, A.; Ayed, S. (2009). Simulation based throughput assessment of nonhomogeneous transfer lines, International Journal of Simulation Modelling, Vol. 8, No. 1, 5-15

[36] Conway, R. W. (1963). Some tactical problems in digital simulation, Management Science, Vol. 10, No. 1, 47-61

[37] Stecke, K. E.; Solberg, J. J. (1981). Loading and control policies for a flexible manufacturing system, International Journal of Production Research, Vol. 19, No. 5, 481-490

[38] Neter, J.; Wasserman, W.; Kutner, M. (1985). Applied linear statistical models - regression, analyses of variance, and experimental designs, Richard D. Irwin, Homewood, Illinois

[39] Eisenhart, C. (1947). The assumptions underlying the analysis of variance, Biometrics, Vol. 3, No. $1,1-21$ 